\title{
Clinicopathological and molecular features of responders to nivolumab for patients with advanced gastric cancer
}

\author{
Saori Mishima ${ }^{1,2+}$, Akihito Kawazoe ${ }^{1 \dagger}$, Yoshiaki Nakamura ${ }^{1}$, Akinori Sasaki ${ }^{1}$, Daisuke Kotani ${ }^{1,2}$, Yasutoshi Kuboki ${ }^{1}$, \\ Hideaki Bando', Takashi Kojima', Toshihiko Doi ${ }^{1}$, Atsushi Ohtsu', Takayuki Yoshino', Takeshi Kuwata², \\ Akihito Tsuji $^{2}$ and Kohei Shitara ${ }^{1 *}$
}

\begin{abstract}
Background: Clinicopathological and molecular features of responders to nivolumab for advanced gastric cancer (AGC) are not well understood.

Methods: Patients (pts) with AGC who were treated with nivolumab after two or more chemotherapy regimens in a single institution from September 2017 to May 2018 were enrolled in this study. PD-L1 expression in tumor cells (TC) and mismatch repair (MMR) were analyzed by immunohistochemistry. Epstein-Barr virus (EBV) was detected by in situ hybridization. Cancer genome alterations were evaluated by a next-generation sequencing-based panel. High tumor mutation burden (TMB) was defined as more than 10 mutations/megabase.

Results: A total of 80 pts were analyzed in this study. Tumor response was evaluated in 72 pts with measurable lesions and 14 pts (19\%) had an objective response. Overall response rate (ORR) was significantly higher in pts with ECOGPS 0 in those with PS 1 or 2, MMR-deficient (MMR-D) in those with MMR-proficient (MMR-P), PD-L1+ in TC in those with PD-L1- in TC and PIK3CA mutation in those with PIK3CA wild-type. ORR was 31\% in pts with at least one of the following factors; MMR-D, high TMB, EBV+ and PD-L1+ in TC vs. 0\% in those without these factors.

Progression-free survival was significantly longer in pts with PS 0 than in those with PS 1 or 2, MMR-D than in those with MMR-P, and PD-L1+ in TC than in those with PD-L1- in TC.
\end{abstract}

Conclusions: Some features were associated with favorable response to nivolumab for AGC. Combining these features might be useful to predict efficacy.

Keywords: Nivolumab, PD-1 inhibitor, Predictive factor, Gastric cancer, Responders

\section{Introduction}

Recently, blockade of immune checkpoint molecules with monoclonal antibodies has emerged as a promising strategy in several malignancies [1-6]. Programmed death 1 (PD-1), which belongs to the $\mathrm{CD} 28$ family of proteins, is a negative costimulatory receptor expressed on the surfaced of activated $\mathrm{T}$ cells [7]. The binding of $\mathrm{PD}-1$ and its ligands, PD-L1 and PD-L2 in tumor or immune cells, can inhibit a cytotoxic T-cell response, which leads tumor cells to escape

\footnotetext{
* Correspondence: kshitara@east.ncc.go.jp

† Saori Mishima and Akihito Kawazoe contributed equally to this work ${ }^{1}$ Department of Gastroenterology and Gastrointestinal Oncology, National Cancer Center Hospital East, 6-5-1 Kashiwanoha, Kashiwa 277-8577, Japan Full list of author information is available at the end of the article
}

from immune surveillance [7]. Accordingly, blockade of this interaction restores the antitumor activity of $\mathrm{T}$ cells [7]. Clinical trials of anti-PD-1/PD-L1 monoclonal antibodies have shown durable anti-tumor response and improved overall survival in several malignancies [1-6].

A phase III ATTRACTION-2 trial of nivolumab, a fully human IgG4 monoclonal antibody (mAb) against PD-1, for patients (pts) with advanced gastric cancer (AGC) after two or more previous line chemotherapies showed a survival benefit, leading to the approval of nivolumab for AGC in Japan [8]. Exploratory analysis of ATTRACTION-2 suggested a survival benefit of nivolumab regardless of PD-L1 expression on tumor cells, thus

(c) The Author(s). 2019 Open Access This article is distributed under the terms of the Creative Commons Attribution 4.0 International License (http://creativecommons.org/licenses/by/4.0/), which permits unrestricted use, distribution, and reproduction in any medium, provided you give appropriate credit to the original author(s) and the source, provide a link to the Creative Commons license, and indicate if changes were made. The Creative Commons Public Domain Dedication waiver (http://creativecommons.org/publicdomain/zero/1.0/) applies to the data made available in this article, unless otherwise stated. 
nivolumab have been used without any restriction by biomarkers [8].

Pembrolizumab, another PD-1 mAb, also demonstrated encouraging anti-tumor activity with acceptable safety for PD-L1 positive AGC in phase II and III trials $[9,10]$, where PD-L1 expression has been evaluated as combined positive score (CPS) counting both tumor cells and immune cells. A relationship between greater PD-L1 CPS and a greater treatment effect was suggested in phase II and III trials of pembrolizumab $[9,10]$. ORR in pts with $\mathrm{CPS} \geq 10, \mathrm{CPS} \geq 1$, and $\mathrm{CPS}<1$ (PD-L1-) were 25,16 , and $2 \%$, respectively [10]. Recently, the US Food and Drug Administration approved pembrolizumab for pts with microsatellite instability-high or mismatch repair (MMR) deficient solid tumors including AGC based on the durable response in several trials [11-13]. In addition to PD-L1 expression and MMR deficiency, a small study suggested that high tumor mutation burden (TMB) and EBV positive status were associated with response to pembrolizumab [14]. However, predictive factors of nivolumab for AGC have not yet been evaluated. Also, overlapping of several clinicopathological and molecular features have not yet been discussed in detail.

In order to establish the better selection of pts who may derive greater benefit from PD-1 blockade, we investigated clinicopathological and molecular features of responders to nivolumab for AGC.

\section{Patients and method \\ Patients}

A prospective study was performed to evaluate the efficacy of nivolumab in pts with AGC from September 2017 to May 2018 at the National Cancer Center Hospital East. The eligibility criteria were the presence of histologically proven adenocarcinoma; Eastern Cooperative Oncology Group performance status (ECOG PS) of $0-2$; adequate bone marrow, hepatic, and renal function; history of previous treatment with two or more regimens and at least one treatment with nivolumab. All patients provided written, informed consent prior to participating in this observational study. The study protocol was approved by the Institutional Review Board at the National Cancer Center.

\section{Molecular characteristics}

Molecular characteristics, such as status of human epidermal growth factor receptor 2 (HER2), PD-L1, MMR, and $\mathrm{EBV}$, and genomic alterations, were analyzed with forma lin-fixed paraffin-embedded tissue specimens from archival tissue samples if available. Immunohistochemistry (IHC) using a monoclonal anti-HER2 antibody (PATHWAY HER2 [4B5], Ventana, Tucson, AZ) and fluorescence in situ hybridization (FISH) using the PathVysion
HER-2 probe kit (Abbott Laboratories, Abbott Park, IL) were performed to assess HER2 status, and HER2 positive was defined as IHC $3+$ or IHC $2+$ and FISH positive. PD-L1 IHC was performed using an anti-PD-L1 rabbit monoclonal antibody (Clone SP142 or SP263, Ventana), and PD-L1 positive in tumor cells (TC) or immune cells (IC) was defined as the presence of $\geq 1 \%$ of TC or IC with membrane staining. CPS, which was the number of PD-L1 staining cells (TC, lymphocytes, and macrophages) divided by the total number of viable TC multiplied by 100 , was also assessed. MMR status was assessed by IHC using monoclonal antibodies for anti-mutL homolog 1 (MLH1, ES05), anti-mutS homolog 2 (MSH2, FE11), anti-postmeiotic segregation increased 2 (PMS2, EP51), and anti-mutS homolog 6 (MSH6, EP49) (Agilent Technologies, Santa Clara, CA), and tumors lacking either MLH1, MSH2, PMS2, or MSH6 expression were considered MMR-deficient (MMR-D), whereas tumors that maintained expression of MLH1, MSH2, PMS2, and MSH6 were considered MMR proficient (MMR-P). Chromogenic in situ hybridization for EBV-encoded RNA (EBER) using fluorescein-labeled oligonucleotide probes (INFORM EBER Probe, Ventana) was performed to assess EBV status [15]. All the specimens were reviewed by TK for this study. Genomic alterations were assessed using Oncomine $^{\mathrm{mu}}$ Comprehensive Assay version 3 or Oncomine $^{\text {Tu }}$ Cancer Research Panel (Thermo Fisher Scientific, Waltham, MA), which allows to detect gene mutations, copy number variants and fusions across multiple genes (Additional file 1: Table S1). TMB was defined as the number of non-synonymous mutations, including indel, per megabase $(\mathrm{mt} / \mathrm{Mb})$ of genome examined in tumor tissue. Known germline variants in dbSNP and East Asian population of 1000 Genomes or ExAC database were not counted. High TMB was defined as more than 10 mutations per megabase.

\section{Outcomes and statistical analysis}

We assessed ORR, disease control rate (DCR), and pro gression-free survival (PFS). Tumor response was assessed in pts with measurable lesions according to the guidelines of the Response Evaluation Criteria in Solid Tumors version 1.1. ORR was defined as the proportion of pts with the best overall response of complete response (CR) or partial response (PR). DCR was defined as the proportion of pts with the best overall response of CR, PR, or stable disease (SD). Responder was defined as pts who achieved $\mathrm{CR}$ or $\mathrm{PR}$, while non-responder was defined as those who showed SD or progression disease (PD). PFS was defined from the date of initiation of nivolumab to the date of disease progression or death from any cause.

Statistical comparisons of ORR according to baseline characteristics was performed using Chi-square test or 
Fisher's exact test. PFS was estimated by the KaplanMeier method and compared according to baseline characteristics using Cox proportional hazards models and presented as hazard ratios (HRs) with 95\% confidence intervals (CIs). Multivariate analysis for PFS was conducted using clinical factors which were associated with significant impact on PFS as well as potential predictive biomarkers reported in previous studies. Statistical analyses were performed using SPSS ${ }^{\circ}$ Statistics software V21 (IBM, Armonk, NY, US). All tests were two-sided; $p<$ 0.05 was considered to indicate statistical significance.

\section{Results}

\section{Patient characteristics}

A total of 80 pts were analyzed in this study. Baseline patient characteristics were shown in Additional file 2: Table S2. The median age was 67 (range, 25-86) years, and 61 pts $(76 \%)$ were male. Forty-seven pts (59\%) had an ECOG PS of 0, whereas the remaining 33 pts (41\%) had a PS of 1 or 2 at the initiation of nivolumab treatment. Forty-nine pts $(61 \%)$ had been treated with three or more lines of previous chemotherapies before nivolumab treatment. The most common metastatic site was the lymph node (75\%), followed by the peritoneum and liver. Sixteen pts (20\%) showed HER2-positive tumors. Eight pts (10\%) were found to have MMR-D status, and 4 (5\%) pts showed EBV-positive tumors.

\section{Clinicopathological and molecular features of responders} to nivolumab

Of 80 pts with AGC, tumor response was evaluated in 72 pts with measurable lesions. Best responses were $C R$, PR, SD, and PD in 0 (0\%), 14 (19\%), 20 (28\%), and 38 (53\%), respectively, resulting in ORR of $19 \%$ and DCR of $47 \%$.

ORR was significantly higher in pts with PS of 0 than in those with PS of 1 or $2(30 \%$ vs. $3 \%, p<0.01)$ (Table 1$)$. There were no other clinical factors significantly associated with responders. ORR tended to be higher in pts with lymph node metastasis than in those without $(24 \%$ vs. $0 \%, p=0.05$ ), although the differences were not statistically significant.

ORR was significantly higher in pts with MMR-D than in those with MMR-P (75\% vs. $13 \%, p<0.01)$, PD-L1+ in TC than in those with PD-L1- in TC $(57 \%$ vs. $13 \%, p<$ 0.01 ), and PIK3CA mutation in those with PIK3CA wild-type ( $44 \%$ vs. $14 \%, p=0.03$ ) (Table 2 ). There were no other molecular factors significantly associated with responders. Additional file 3: Figure S1 also showed no significant relationship between $\mathrm{TMB}$ and response to nivolumab. ORR in pts with CPS $\geq 10, \mathrm{CPS} \geq 1, \mathrm{CPS}<1$ (PD-L1-), EBV+ and high TMB were 35, 26, 0, 25, and $22 \%$, respectively. After excluding 8 pts with MMR-D from the analysis, PS of 0 and PD-L1+ in TC were factors significantly associated with responders in 60 pts with MMR-P (Additional file 4: Table S3 and Additional file 5: Table S4).

Table 1 Clinical features of responders to nivolumab

\begin{tabular}{|c|c|c|c|c|c|c|}
\hline$n=72$ & & All & Responder & Non-responder & ORR & $P$-value \\
\hline \multirow[t]{2}{*}{$\overline{\text { Age }}$} & $<65$ & $26(36 \%)$ & 8 & 18 & $31 \%$ & 0.07 \\
\hline & $\geq 65$ & $46(64 \%)$ & 6 & 40 & $13 \%$ & \\
\hline \multirow[t]{2}{*}{ Gender } & Male & $56(78 \%)$ & 11 & 45 & $20 \%$ & 0.94 \\
\hline & Female & $16(22 \%)$ & 3 & 13 & $19 \%$ & \\
\hline \multirow[t]{2}{*}{ ECOG PS } & 0 & $43(60 \%)$ & 13 & 30 & $30 \%$ & $<0.01$ \\
\hline & $\geq 1$ & $29(40 \%)$ & 1 & 28 & $3 \%$ & \\
\hline \multirow[t]{2}{*}{ Histology } & Intestinal & $31(43 \%)$ & 5 & 26 & $16 \%$ & 0.54 \\
\hline & Diffuse & $41(57 \%)$ & 9 & 32 & $22 \%$ & \\
\hline \multirow[t]{2}{*}{ Borrmann classification } & Type4 & $7(10 \%)$ & 0 & 7 & $0 \%$ & 0.17 \\
\hline & Others & 65 (90\%) & 14 & 51 & $22 \%$ & \\
\hline \multirow[t]{2}{*}{ Number of previous chemotherapy } & 2 & $29(40 \%)$ & 8 & 21 & $28 \%$ & 0.15 \\
\hline & $\geq 3$ & $43(60 \%)$ & 6 & 37 & $14 \%$ & \\
\hline \multirow[t]{4}{*}{ Site of metastasis } & Lymph node & $59(82 \%)$ & 14 & 45 & $24 \%$ & 0.05 \\
\hline & Peritoneum & $35(49 \%)$ & 5 & 30 & $14 \%$ & 0.28 \\
\hline & Liver & $32(44 \%)$ & 4 & 28 & $13 \%$ & 0.18 \\
\hline & Lung & $10(14 \%)$ & 1 & 9 & $10 \%$ & 0.42 \\
\hline \multirow[t]{2}{*}{ Number of metastatic sites } & 1 & $21(29 \%)$ & 4 & 17 & $19 \%$ & 0.96 \\
\hline & $\geq 2$ & $51(71 \%)$ & 10 & 41 & $20 \%$ & \\
\hline
\end{tabular}


Table 2 Molecular features of responders to nivolumab

\begin{tabular}{|c|c|c|c|c|c|c|}
\hline & Assessed & Detected & Responder & Non-responder & ORR & $P$-value \\
\hline HER2+ & 71 & $16(23 \%)$ & 1 & 15 & $6 \%$ & 0.12 \\
\hline PD-L1+ in tumor cell & 60 & $14(23 \%)$ & 8 & 6 & $57 \%$ & $<0.01$ \\
\hline$C P S \geq 10$ & 60 & $17(28 \%)$ & 6 & 11 & $35 \%$ & 0.17 \\
\hline$C P S \geq 1$ & 60 & $54(90 \%)$ & 14 & 40 & $26 \%$ & 0.15 \\
\hline EBV+ & 68 & $4(6 \%)$ & 1 & 3 & $25 \%$ & 0.82 \\
\hline MMR-D & 68 & $8(12 \%)$ & 6 & 2 & $75 \%$ & $<0.01$ \\
\hline$T M B \geq 10$ & 54 & $32(59 \%)$ & 7 & 25 & $22 \%$ & 0.44 \\
\hline ARIDIA mutation & 52 & $5(10 \%)$ & 1 & 4 & $25 \%$ & 0.96 \\
\hline ERBB2 mutation & 52 & $2(4 \%)$ & 0 & 2 & $0 \%$ & 0.48 \\
\hline KRAS mutation & 52 & $4(8 \%)$ & 0 & 4 & $0 \%$ & 0.31 \\
\hline MET mutation & 52 & $2(4 \%)$ & 0 & 2 & $0 \%$ & 0.48 \\
\hline PIK3CA mutation & 52 & $9(17 \%)$ & 4 & 5 & $44 \%$ & 0.03 \\
\hline TP53 mutation & 52 & $28(54 \%)$ & 6 & 22 & $21 \%$ & 0.66 \\
\hline CCNE1 amplification & 52 & $7(13 \%)$ & 2 & 5 & $29 \%$ & 0.50 \\
\hline ERBB2 amplification & 52 & $9(17 \%)$ & 0 & 9 & $0 \%$ & 0.11 \\
\hline FGFR amplification & 52 & $3(6 \%)$ & 0 & 3 & $0 \%$ & 0.38 \\
\hline MDM2 amplification & 52 & $2(4 \%)$ & 0 & 2 & $0 \%$ & 0.48 \\
\hline MYC amplification & 52 & $3(6 \%)$ & 0 & 3 & $0 \%$ & 0.38 \\
\hline
\end{tabular}

CPS combined positive score, EBV Epstein-Barr virus, MMR-D mismatch repair deficient, ORR objective response rate, PD-L1 programmed cell death-1 ligand-1, TMB tumor mutation burden

Table 3 Characteristics of patients with response to nivolumab

\begin{tabular}{|c|c|c|c|c|c|c|c|c|c|}
\hline \multirow[t]{2}{*}{ Age } & \multirow[t]{2}{*}{ PS } & \multicolumn{3}{|l|}{ Genomic alteration } & \multirow{2}{*}{$\begin{array}{l}\text { PD- } \\
\mathrm{L} 1 \\
+ \\
\text { in } \\
\mathrm{TC}\end{array}$} & \multirow[t]{2}{*}{$C P S \geq 10$} & \multirow[t]{2}{*}{$C P S \geq 1$} & \multirow[t]{2}{*}{ EBV } & \multirow[t]{2}{*}{ MMR } \\
\hline & & Mutation & Amplification & $\mathrm{TMB} / \mathrm{Mb}$ & & & & & \\
\hline 63 & 0 & $\mathrm{NE}$ & $\mathrm{NE}$ & $\mathrm{NE}$ & - & + & + & - & MMR-D \\
\hline 63 & 0 & $\mathrm{NE}$ & $\mathrm{NE}$ & $\mathrm{NE}$ & + & + & + & - & MMR-D \\
\hline 66 & 0 & PIK3CA, TP53 & None & 38.3 & + & - & + & - & MMR-D \\
\hline 62 & 0 & PIK3CA & None & 11.5 & - & - & + & - & MMR-D \\
\hline 53 & 1 & None & None & 7.7 & + & + & + & - & MMR-D \\
\hline 79 & 0 & MET, PIK3CA, TP53 & None & 58.0 & + & - & + & - & MMR-D \\
\hline 64 & 0 & PIK3CA & None & 15.3 & + & + & + & - & MMR-P \\
\hline 74 & 0 & ARID1A, TP53 & CCNE1 & 15.1 & - & - & + & - & MMR-P \\
\hline 80 & 0 & TP53 & CCNE1 & 11.5 & - & - & + & - & MMR-P \\
\hline 76 & 0 & None & None & 10.1 & - & - & + & - & MMR-P \\
\hline 73 & 0 & TP53 & None & 5.0 & + & + & + & - & MMR-P \\
\hline 65 & 0 & $\mathrm{NE}$ & $\mathrm{NE}$ & $\mathrm{NE}$ & + & + & + & - & MMR-P \\
\hline 53 & 0 & $\mathrm{NE}$ & $\mathrm{NE}$ & $\mathrm{NE}$ & + & - & + & - & MMR-P \\
\hline 43 & 0 & TP53 & None & 7.7 & - & - & + & + & MMR-P \\
\hline
\end{tabular}


Table 3 showed characteristics of pts with response to nivolumab. Among the 14 responders, 6 were MMR-D and other 8 were MMR-P. TMB was assessed in 4 MMR-D pts., and 3 of them were with high TMB (range 11.5 to 58.0). Four MMR-P responders were also associated with high TMB (range 10.1 and 15.3). One MMR-P responder was EBV+ with TMB of 7.7 and the remaining 3 MMR-P responders were PD-L1+ in TC. Among MMR-D or EBV+ pts., no EBV+ pts showed PD-L1+ in TC or CPS $\geq 10$. Two patients with MMR-D without tumor response had PS of 1 or PS of 2 as well as PIK3CA mutations (Additional file 6: Table S5).

Importantly, ORR was $31 \%$ in pts with at least one of the following factors; MMR-D, high-TMB, EBV+, and PD-L1+ in TC vs. $0 \%$ in those without these factors.

\section{Progression free survival analysis}

In 80 pts with AGC, the median PFS of nivolumab was 1.9 (95\% CI, 1.5-2.4) months with median follow-up period of 3.8 months (range, 0.3-8.0 months) (Fig. 1a). Subgroup analysis of PFS was shown in Additional file 7: Table S6. PFS was significantly longer in pts with PS of 0 than in those with PS of 1 or 2 (median 3.0 months vs. 1.1 months, HR 0.30 ; 95\% CI $0.18-0.52, p<0.01$ ) (Fig. 1b), MMR-D than MMR-P (median not reached vs. 1.8 months, HR 0.21; 95\% CI 0.06-0.70, $p<0.01$ ) (Fig. 1c), and PD-L1+ in TC than PD-L1- in TC (median not reached vs. 1.9 months, $\mathrm{HR} 0.42 ; 95 \%$ CI $0.19-0.96, p=$ 0.03) (Fig. 1d). In univariate analysis for PFS, PS was only clinical factor associate with PFS. Furthermore, after adjusted by PS, the HR for PFS of pts with MMR-D compared to those with MMR-P and pts with PD-L1+ in TC compared to those with PD-L1in TC was $0.2(95 \%$ CI $0.1-0.6 ; p<0.01)$ and $0.4(95 \%$ CI $0.2-0.9 ; \mathrm{p}=0.03$ ), respectively (Additional file 7 : Table S6). Pts with other molecular features associated with response to nivolumab in this study did not show significantly longer PFS.
A Progression free survival

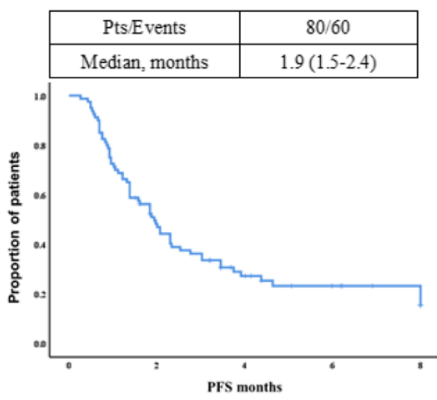

B Progression free survival by PS

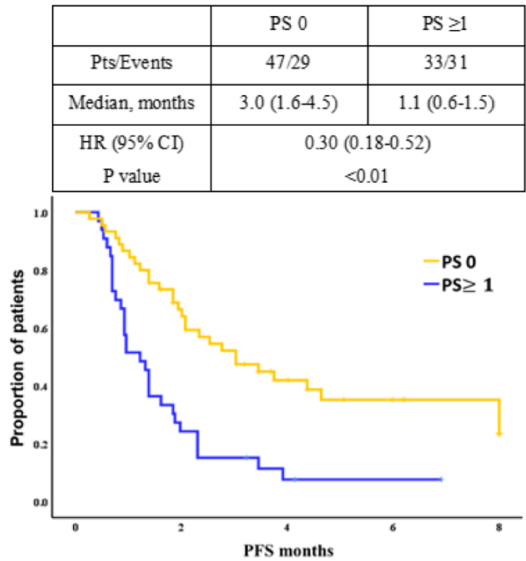

Progression free survival by MMR status

\begin{tabular}{|c|c|c|}
\hline & MMR-D & MMR-P \\
\hline Pts/Events & $8 / 3$ & $66 / 52$ \\
\hline Median, months & N.R. & $1.8(1.3-2.4)$ \\
\hline HR (95\% CD) & \multicolumn{2}{|c|}{$0.21(0.06-0.70)$} \\
P value & \multicolumn{2}{|c|}{$<0.01$} \\
\hline
\end{tabular}

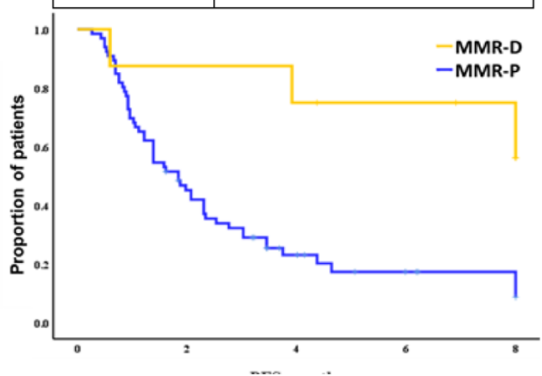

D

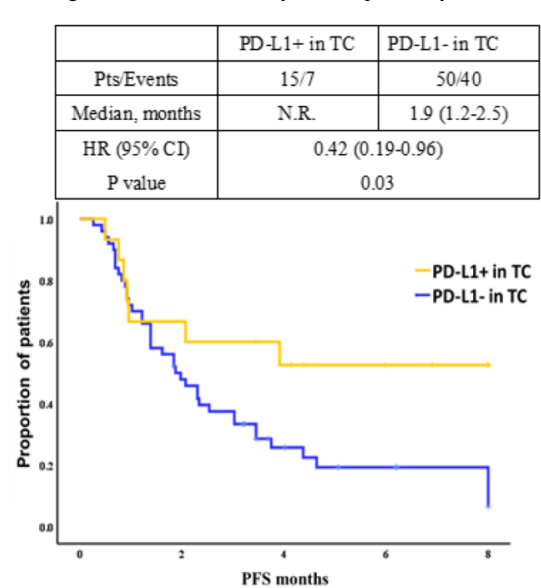

Fig. 1 Progression free survival. a Progression free survival. b Progression free survival by PS. c Progression free survival by MMR status. d Progression free survival by PD-L1 positivity in tumor cell. MMR, mismatch repair; MMR-D, mismatch repair deficient; MMR-P, mismatch repair proficient; PD-L1, programmed cell death-1 ligand-1; PS, Eastern Cooperative Oncology Group performance status; Pts, patient 


\section{Discussion}

In this study, we investigated the characteristics of responders to nivolumab for pts with AGC. To our knowledge, this is the first report to provide detailed information on clinicopathological and molecular features associated with response to nivolumab for AGC.

The results of subgroup analysis of phase II and III trials of pembrolizumab showed that better PS was associated with a higher response rate and longer overall survival $[10,11]$. Consistent with these results, pts with PS of 0 had better ORR and PFS compared to those with PS of 1 or 2 in our study. Furthermore, after excluding pts with MMR-D from the analysis, PS of 0 was an only clinical factor significantly associated with responders in pts with MMR-P, suggesting that it is important to assess general condition before the initiation of PD-1 blockade for the prediction of efficacy. Although the exact explanations for the correlation between PS and clinical outcomes of PD-1 blockade were not established, pts with poor PS may not stay on treatment long enough to achieve a response.

In our analysis, PD-L1 expression in TC was significantly associated with responders to nivolumab for AGC, which was contrary to that of subgroup analysis from ATTRACTION-2 [8]. Furthermore, after excluding pts with MMR-D, impact of PD-L1 in TC was still statistically significant. Different anti-PD-L1 antibodies (SP142 or SP263) in our study from those (28-8 or $22 \mathrm{C} 3)$ in these previous studies of nivolumab or pembrolizumab $[9,10,14]$ might affect the predictive value of PD-L1 expression. Also, ORR and PFS tended to be better in pts with CPS $\geq 10$ overlapping substantially with PD-L1+ in TC in our analysis; 5 of 14 responders had both CPS $\geq 10$ and PD-L1+ in TC. Impact of CPS on the efficacy of PD-1 blockade will also be evaluated in the ongoing phase III KEYNOTE062 trial (NCT02494583), which compared the efficacy of cytotoxic agents combined to pembrolizumab with that of cytotoxic agents and that of pembrolizumab monotherapy in pts with untreated AGC.

ORR was significantly higher in AGC pts with PIK3CA mutation in our study, which was consistent with a recent study analyzing genomic correlates of response to immune checkpoint blockade in microsatellite-stable solid tumors [16]. It is also suggested that PIK3CA mutation have been linked with APOBEC signatures which is highly proficient at generating DNA breaks whose repair can trigger the formation of single-strand hypermutation substrates [17]. Moreover, in gastric cancer, it has been well known that APOBEC -mutation signature and PIK3CA mutation were frequently observed in EBV+ pts [18]. Meanwhile, it is reported that PIK3CA mutation is strongly associated with the MSI molecular subgroup [19]. Among 4 responders with PIK3CA mutation in our study, 3 were MMR-D, and only additional one patient with MMR-P, no EBV+, and PD-L1 in TC with CPS $\geq 10$ had mutation in PIK3CA lie in E542K, which has been reported to be associated with APOBEC signature. Thus, the predictive value of PIK3CA mutation alone in AGC needs further investigations. Most recently, extremely high ORR (100\%) of pembrolizumab was reported in 6 pts with EBV+ AGC [14], which was inconsistent with our result showing that 1 of 4 pts with EBV+ (25\%) achieved an objective response. Notably, no EBV pts in our study showed CPS $\geq 10$, which was different from previous study [14]. Our pervious study showed not all EBV+ tumors showed high PD-L1 expression [15], thus both EBV status and PD-L1 expression should be evaluated in a larger cohort.

High TMB was not associated with response to nivolumab in our study, though it was notable that 4 of 8 responder pts with MMR-P had high TMB. It has been reported that the estimated TMB based on panel sequencing showed relatively high discordance compared with TMB calculated from whole exome sequencing in tumors with relatively low number of mutations [20], which might lead to the results in this study which did not show good correlation between anti-tumor response and TMB. Recently, Kim ST et al. reported that high TMB was a potential biomarker of pembrolizumab for AGC [14]. However, most pts with high TMB had MMR-D status, and not all pts with high TMB achieved an objective response [14]. Thus, precise mechanism regarding the influence of TMB to the efficacy of PD-1/ PD-L1 blockade should be investigated in the near future.

Interestingly, ORR was $31 \%$ in pts with at least one of the following factors; MMR-D, high-TMB, EBV+, and $\mathrm{PD}-\mathrm{L} 1+$ in $\mathrm{TC}$ vs. $0 \%$ in those without these factors, suggesting that pre-screening of these biomarkers might be useful to predict clinical benefit of anti-PD-1/PD-L1 blockade in AGC.

It is important to note the limitations of the present study. First, this was a single-institution study with a limited sample size. Second, we did not analyze PD-L1 expression, MMR, EBV status, and cancer genome alterations in all the pts enrolled in this study, which warrants further evaluations in a larger cohort.

In conclusion, we identified some clinicopathological and molecular characteristics associated with responders to nivolumab for pts with AGC. Combining these features might be useful for the better selection of pts who may derive greater benefit from PD-1 blockade. However, further investigations in larger cohorts are needed to confirm precise biomarkers of PD-1/PD-L1 blockade for AGC. 


\section{Additional files}

Additional file 1: Table S1. Gene list of the Oncomine ${ }^{\mathrm{TM}}$

Comprehensive Assay version 3. (DOCX $15 \mathrm{~kb}$ )

Additional file 2: Table S2. Patient characteristics. (DOCX $17 \mathrm{~kb}$ )

Additional file 3: Figure S1. Response to nivolumab by tumor mutation burden. (DOCX $22 \mathrm{~kb}$ )

Additional file 4: Table S3. Clinicopathological features of responders to nivolumab in patients with MMR-P. (DOCX $16 \mathrm{~kb}$ )

Additional file 5: Table S4. Molecular features of responders to nivolumab in patients with MMR-P. (DOCX $16 \mathrm{~kb}$ )

Additional file 6: Table S5. Characteristics of patients with MMR-D and EBV positive gastric cancer. (DOCX $18 \mathrm{~kb}$ )

Additional file 7: Table S6. Subgroup analysis of progression-free survival. (DOCX $16 \mathrm{~kb}$ )

\section{Abbreviations \\ AGC: Advanced gastric cancer; Cls: Confidence intervals; CPS: Combined positive score; CR: Complete response; DCR: Disease control rate; EBER: EBV- encoded RNA; EBV: Epstein-Barr virus; ECOG PS: Eastern Cooperative Oncology Group performance status; FISH: Fluorescence in situ hybridization; HER2: Human epidermal growth factor receptor 2; HR: Hazard ratio; IC: Immune cell; IHC: Immunohistochemistry; mAbs: Monoclonal antibodies; MLH1: Anti-mutL homolog 1; MMR: Mismatch repair; MMR-D: Mismatch repair deficient; MMR-P: Mismatch repair proficient; MSH2: Anti-mutS homolog 2; MSH6: Anti-mutS homolog 6; mt/MB: Mutations/megabase; ORR: Objective response rate; PD: Progressive disease; PD-1: Anti- programmed death-1; PD-L1: Programmed death-ligand 1; PD- L2: Programmed death-ligand 2; PFS: Progression-free survival; PMS2: Anti- postmeiotic segregation increased 2; PR: Partial response; pts.: Patients; SD: Stable disease; TC: Tumor cell; TMB: Tumor mutation burden}

\section{Acknowledgements}

None declared

\section{Funding}

This study was supported by a research funding from National Cancer Center Hospital East (none apply).

\section{Availability of data and materials}

All data analyzed during this study has been included within the article.

\section{Author's contributions}

SM, AK, YN, SA and KS designed the study, collected data, performed data analysis and wrote manuscript. DK, YK, HB, TK, TD, AO, and AT were involved in data interpretation and critically reviewing the manuscript. TK was involved in testing tumor tissue as well as critically reviewing the manuscript. All authors read and approved the final manuscript.

\section{Ethics approval and consent to participate}

All procedures followed in this study were in accordance with the Declaration of Helsinki of 1964 and later versions and the Japanese Ethical Guidelines for Medical and Health Research Involving Human Subjects. Informed consent for it was obtained from all patients for their being included in the study.

\section{Consent for publication}

This is not applicable for this study.

\section{Competing interests}

SM has nothing to disclose.

AK reports research funding from Ono, Sumitomo Dainippon, and Taiho.

AS has nothing to disclose.

YN reports personal fees from Chugai.

DK has nothing to disclose.

YK reports consulting or advisory role for Takeda; personal fees from Bayer, Lilly, and Taiho; and research funding from Astra Zeneca, Daiichi Sankyo, Incyte, Taiho, and Takeda.
$\mathrm{HB}$ reports research funding from Astra Zeneca and Sysmex.

TK reports personal fees from MSD; and research funding from Astellas, Bristol-Myers Squibb, MSD, Oncolys BioPharma, Ono, Shionogi. TD reports consulting or advisory role for Amgen, Chugai, Daiichi Sankyo, Kyowa Hakko Kirin, Lilly, MSD, Sumitomo Dainippon, and Taiho; and research funding from Abbvie, Astellas, Boehringer Ingelheim, Bristol-Myers Squibb, Celgene, Chugai, Daiichi Sankyo, Janssen, Kyowa Hakko Kirin, Lilly, Merck Serono, MSD, Novartis, Pfizer, Quintiles, Sumitomo Group, Takeda, and Taiho. AO reports personal fees from Bristol-Myers Squibb, Chugai, Ono, and Taiho. TY consulting or advisory role for Chugai, Lilly, Merck Serono, and Sanofi; and research funding from Chugai, GlaxoSmithKline, MSD, Nippon Boehringer Ingelheim, Sanofi, and Sumitomo Dainippon

TK consulting or advisory role for ThermoFisher Inc.; personal fees from Chugai and Roche Diagnostics; and research funding from Daiichi-Sankyo. AT reports personal fees from Bristol-Myers Squibb, Chugai, Daiichi Sankyo, Merck Serono, Taiho, and Takeda.

KS reports consulting or advisory role for Astellas, Bristol-Myers Squibb, Lilly, Ono, Pfizer, and Takeda; personal fees from AbbVie, Novartis, and Yakult; and research funding from Chugai, Daiichi Sankyo, Lilly, MSD, Ono, Sumitomo Dainippon, and Taiho.

\section{Publisher's Note}

Springer Nature remains neutral with regard to jurisdictional claims in published maps and institutional affiliations.

\section{Author details}

${ }^{1}$ Department of Gastroenterology and Gastrointestinal Oncology, National Cancer Center Hospital East, 6-5-1 Kashiwanoha, Kashiwa 277-8577, Japan. ${ }^{2}$ Graduated School of Medicine, Kagawa University, Takamatsu, Japan. ${ }^{3}$ Department of Pathology and Clinical Laboratories, National Cancer Center Hospital East, Kashiwa, Japan.

Received: 16 October 2018 Accepted: 20 January 2019 Published online: 31 January 2019

\section{References}

1. Topalian SL, Hodi FS, Brahmer JR, Gettinger SN, Smith DC, McDermott DF, et al. Safety, activity, and immune correlates of anti-PD-1 antibody in cancer. N Engl J Med. 2012;366(26):2443-54.

2. Robert C, Long GV, Brady B, Dutriaux C, Maio M, Mortier L, et al. Nivolumab in previously untreated melanoma without BRAF mutation. N Engl J Med. 2015:372(4):320-30.

3. Reck M, Rodríguez-Abreu $D$, Robinson AG, Hui R, Csőszi T, Fülöp $A$, et al. Pembrolizumab versus chemotherapy for PD-L1-positive non-small-cell lung Cancer. N Engl J Med. 2016;375(19):1823-33.

4. Herbst RS, Baas P, Kim DW, Felip E, Pérez-Gracia JL, Han JY, et al. Pembrolizumab versus docetaxel for previously treated, PD-L1-positive, advanced non-small-cell lung cancer (KEYNOTE-010): a randomised controlled trial. Lancet. 2016;387(10027):1540-50.

5. Charles SF, Doi T, Raymond WJJ, Muro K, Satoh T, Machado M, et al. KEYNOTE-059 cohort 1: efficacy and safety of pembrolizumab (pembro) monotherapy in patients with previously treated advanced gastric cancer. J Clin Oncol. 2017;35(15_suppl):4003.

6. Motzer RJ, Escudier B, McDermott DF, George S, Hammers HJ, Srinivas S, et al. Nivolumab versus Everolimus in advanced renal-cell carcinoma. N Engl J Med. 2015;373(19):1803-13.

7. Pardoll DM. The blockade of immune checkpoints in cancer immunotherapy. Nat. Rev Cancer. 2012;12(4):252-64.

8. Kang YK, Boku N, Satoh T, Ryu MH, Chao Y, Kato K, et al. Nivolumab in patients with advanced gastric or gastro-oesophageal junction cancer refractory to, or intolerant of, at least two previous chemotherapy regimens (ONO-4538-12, ATTRACTION-2): a randomised, double-blind, placebocontrolled, phase 3 trial. Lancet. 2017:390(10111):2461-71.

9. Fuchs CS, Doi T, Jang RW, Muro K, Satoh T, Machado M, et al. Safety and efficacy of Pembrolizumab monotherapy in patients with previously treated advanced gastric and gastroesophageal junction Cancer: phase 2 clinical KEYNOTE-059 trial. JAMA Oncol. 2018;4:e180013.

10. Shitara K, Ozguroglu M, Bang YJ, et al. Pembrolizumab versus paclitaxel for previously treated, advanced gastric or gastro-oesophageal junction cancer (KEYNOTE-061): a randomised, open-label, controlled, phase 3 trial. Lancet. 2018;392:123-33. 
11. Le DT, Uram JN, Wang H, Di Bartolomeo M, Mandalà M, Ryu MH, et al. PD-1 blockade in tumors with mismatch-repair deficiency. N Engl J Med. 2015; 372(26):2509-20

12. Le DT, Durham JN, Smith KN, Wang H, Bartlett BR, Aulakh LK, et al. Mismatch repair deficiency predicts response of solid tumors to PD-1 blockade. Science. 2017;357:409-13.

13. Diaz LA Jr, Marabelle A, Delord JP, Shapira-Frommer R, Geva R, Peled N, et al. Pembrolizumab therapy for microsatellite instability high (MSI-H) colorectal cancer (CRC) and non-CRC. J Clin Oncol. 2017;35(15 suppl):3071.

14. Kim ST, Cristescu R, Bass AJ, Kim KM, Odegaard Jl, Kim K, et al. Comprehensive molecular characterization of clinical responses to PD-1 inhibition in metastatic gastric cancer. Nat Med. 2018; [Epub ahead of print].

15. Kawazoe A, Shitara K, Kuboki Y, Bando H, Kojima T, Yoshino T, et al. Clinicopathological features of 22C3 PD-L1 expression with mismatch repair, Epstein-Barr virus status, and cancer genome alterations in metastatic gastric cancer. Gastric Cancer. 2018; [Epub ahead of print].

16. Miao D, Margolis CA, Vokes NI, Liu D, Taylor-Weiner A, Wankowicz SM, et al. Genomic correlates of response to immune checkpoint blockade in microsatellite-stable solid tumors. Nat Genet. 2018;50(9):1271-81.

17. Henderson S, Chakravarthy A, Su X, Boshoff C, Fenton TR. APOBEC-mediated cytosine deamination links PIK3CA helical domain mutations to human papillomavirus-driven tumor development. Cell Rep. 2014;7(6):1833-41.

18. Cancer Genome Atlas Research Network. Comprehensive molecular characterization of gastric adenocarcinoma. Nature. 2014;513(7517):202-9.

19. Polom K, Marrelli D, Roviello G, Pascale V, Voglino C, Vindigni C, et al. PIK3CA mutation in gastric cancer and the role of microsatellite instability status in mutations of exons 9 and 20 of the PIK3CA gene. Adv Clin Exp Med. 2018;27(7):963-9.

20. Hatakeyama K, Nagashima T, Urakami K, Ohshima K, Serizawa M, Ohnami S, et al. Tumor mutational burden analysis of 2,000 Japanese cancer genomes using whole exome and targeted gene panel sequencing. Biomed Res. 2018;39(3):159-67.

Ready to submit your research? Choose BMC and benefit from:

- fast, convenient online submission

- thorough peer review by experienced researchers in your field

- rapid publication on acceptance

- support for research data, including large and complex data types

- gold Open Access which fosters wider collaboration and increased citations

- maximum visibility for your research: over $100 \mathrm{M}$ website views per year

At $\mathrm{BMC}$, research is always in progress.

Learn more biomedcentral.com/submissions 